\title{
BMJ Global Health Infection and mortality of healthcare workers worldwide from COVID-19: a systematic review
}

Soham Bandyopadhyay (D) , ${ }^{1}$ Ronnie E Baticulon, ${ }^{2}$ Murtaza Kadhum, ${ }^{1}$ Muath Alser, ${ }^{3}$ Daniel K Ojuka, ${ }^{4}$ Yara Badereddin, ${ }^{5}$ Archith Kamath, ${ }^{1}$ Sai Arathi Parepalli, ${ }^{1}$ Grace Brown, ${ }^{1}$ Sara Iharchane,${ }^{6}$ Sofia Gandino, ${ }^{6}$ Zara Markovic-Obiago, ${ }^{1}$ Samuel Scott, ${ }^{1}$ Emery Manirambona, ${ }^{7}$ Asif Machhada, ${ }^{8}$ Aditi Aggarwal, ${ }^{1}$ Lydia Benazaize, ${ }^{1}$ Mina Ibrahim, ${ }^{8}$ David Kim, ${ }^{1}$ Isabel Tol, ${ }^{1}$ Elliott H Taylor, ${ }^{1}$ Alexandra Knighton, ${ }^{1}$ Dorothy Bbaale, ${ }^{9}$ Duha Jasim, ${ }^{1}$ Heba Alghoul, ${ }^{10}$ Henna Reddy, ${ }^{1}$ Hibatullah Abuelgasim, ${ }^{1}$ Kirandeep Saini, ${ }^{1}$ Alicia Sigler, ${ }^{11}$ Leenah Abuelgasim, ${ }^{1}$ Mario Moran-Romero, ${ }^{12}$ Mary Kumarendran, ${ }^{1}$ Najlaa Abu Jamie, ${ }^{13}$ Omaima Ali, ${ }^{1}$ Raghav Sudarshan, ${ }^{1}$ Riley Dean, ${ }^{14}$ Rumi Kisyova, ${ }^{8}$ Sonam Kelzang, ${ }^{15}$ Sophie Roche, ${ }^{1}$ Tazin Ahsan, ${ }^{16}$ Yethrib Mohamed, ${ }^{1}$ Andile Maqhawe Dube, ${ }^{17}$ Grace Paida Gwini, ${ }^{17}$ Rashidah Gwokyala, ${ }^{18,19}$ Robin Brown, ${ }^{1}$ Mohammad Rabiul Karim Khan Papon, ${ }^{16}$ Zoe Li (1) , ${ }^{20}$ Salvador Sun Ruzats, ${ }^{21}$ Somy Charuvila, ${ }^{1}$ Noel Peter, ${ }^{1}$ Khalil Khalidy, ${ }^{10}$ Nkosikhona Moyo, ${ }^{17}$ Osaid Alser (D) , ${ }^{1,22}$ Arielis Solano, ${ }^{23}$ Eduardo Robles-Perez, ${ }^{24}$ Aiman Tariq, ${ }^{25}$ Mariam Gaddah, ${ }^{26}$ Spyros Kolovos, ${ }^{27}$ Faith C Muchemwa, ${ }^{28}$ Abdullah Saleh, ${ }^{29,30}$ Amanda Gosman, ${ }^{29}$ Rafael Pinedo-Villanueva, ${ }^{31,32}$ Anant Jani, ${ }^{1}$ Roba Khundkar ${ }^{1,8}$

To cite: Bandyopadhyay S, Baticulon RE, Kadhum M, et al. Infection and mortality of healthcare workers worldwide from COVID-19: a systematic review. BMJ Global Health 2020;5:e003097. doi:10.1136/ bmjgh-2020-003097

Handling editor Soumyadeep Bhaumik

- Additional material is published online only. To view, please visit the journal online (http://dx.doi.org/10.1136/ bmjgh-2020-003097).

Received 8 June 2020 Revised 19 September 2020 Accepted 9 October 2020

Check for updates

(c) Author(s) (or their employer(s)) 2020. Re-use permitted under CC BY-NC. No commercial re-use. See rights and permissions. Published by BMJ.

For numbered affiliations see end of article.

Correspondence to

Roba Khundkar;

roba.khundkar@nds.ox.ac.uk

\section{ABSTRACT}

Objectives To estimate COVID-19 infections and deaths in healthcare workers (HCWs) from a global perspective during the early phases of the pandemic.

Design Systematic review.

Methods Two parallel searches of academic bibliographic databases and grey literature were undertaken until 8 May 2020. Governments were also contacted for further information where possible. There were no restrictions on language, information sources used, publication status and types of sources of evidence. The AACODS checklist or the National Institutes of Health study quality assessment tools were used to appraise each source of evidence.

Outcome measures Publication characteristics, country-specific data points, COVID-19-specific data, demographics of affected HCWs and public health measures employed.

Results A total of 152888 infections and 1413 deaths were reported. Infections were mainly in women $(71.6 \%$, $\mathrm{n}=14058)$ and nurses $(38.6 \%, \mathrm{n}=10706)$, but deaths were mainly in men $(70.8 \%, \mathrm{n}=550)$ and doctors $(51.4 \%$, $\mathrm{n}=525$ ). Limited data suggested that general practitioners and mental health nurses were the highest risk specialities for deaths. There were 37.2 deaths reported per 100 infections for HCWs aged over 70 years. Europe had the highest absolute numbers of reported infections (119 628) and deaths (712), but the Eastern Mediterranean region had the highest number of reported deaths per 100 infections (5.7).

\section{Key questions}

What is already known?

- In China, studies documented over 3300 confirmed cases of infected healthcare workers in early March.

- In the USA, as high as $19 \%$ of patients had been identified as healthcare workers.

- There are no studies that perform a global examination of COVID-19 infections and deaths in the health workforce.

What are the new findings?

- General practitioners were the highest risk specialty for deaths among doctors, while the highest risk nursing specialty was mental health.

- Most COVID-19 cases and deaths were reported in the 50-59 age range, while the group aged over 70

- Europe had the highest number of infections and deaths, but the lowest case fatality rate, while the Eastern Mediterranean region had the highest case fatality rate; by population, regions such as the Indian subcontinent and Africa reported a relatively low number of infections and deaths.

Conclusions COVID-19 infections and deaths among HCWs follow that of the general population around the world. The reasons for gender and specialty differences require further exploration, as do the low rates reported in years had the highest case fatality rate. 


\section{Key questions}

What do the new findings imply?

- Although physicians working in certain specialties may be considered high-risk due to frequent exposure to oronasal secretions, the risk to other healthcare worker specialties must not be underestimated.

- High rates of morbidity and mortality in elderly healthcare workers may require assigning them to less risky settings such as telemedicine, non-COVID-19 outpatient clinics or administrative positions.

- Furthermore, the disparities between regions of the world may be a true difference or it may reflect reporting and testing bias.

Africa and India. Although physicians working in certain specialities may be considered high risk due to exposure to oronasal secretions, the risk to other specialities must not be underestimated. Elderly HCWs may require assigning to less risky settings such as telemedicine or administrative positions. Our pragmatic approach provides general trends, and highlights the need for universal guidelines for testing and reporting of infections in HCWs.

\section{INTRODUCTION}

From a cluster of patients with pneumonia linked to a wet market in Wuhan, China in late December 2019, COVID-19 has rapidly evolved into a full-blown pandemic. ${ }^{2}$ At the time of writing (23 May), over 5 million people have been infected across 213 countries and territories, leading to $>300000$ deaths worldwide. ${ }^{3}$ On the frontlines of this global crisis are healthcare workers (HCWs) with the substantial task of diagnosing and treating an exponentially growing number of acutely ill patients, often having to make critical decisions under physical and psychological pressure. ${ }^{4-6}$ WHO defines health workers as 'all people engaged in actions whose primary intent is to enhance health'. ${ }^{7}$ This encompasses doctors, nurses, midwives, paramedical staff, hospital administrators and support staff and community workers, all of whom now face the occupational risk of becoming infected with COVID-19, and at worst, even death.

Ensuring the protection of HCWs is a crucial element of any country's strategic response to the COVID-19 crisis, especially as governments rush to increase healthcare capacity to cope with the surge of patients requiring urgent care. WHO has issued recommendations on the rational use of personal protective equipment (PPE) in hospital and community settings. ${ }^{8}$ Several colleges and specialty societies have formulated algorithms and guidelines to decrease the risk of COVID-19 transmission in their fields of practice. ${ }^{9-13}$ Nevertheless, protecting HCWs remains a challenge for most countries, where shortages of adequate PPE is a daily concern. Limited testing capacity precludes early identification and isolation of cases, leading to unnecessary occupational exposure for HCWs, particularly since a high number of patients with COVID-19 remain asymptomatic. In a vicious cycle, shortages of HCWs may compel staff to continue working for days on end, even under fatigue or when symptoms manifest, further increasing the risk of transmission.

Unmitigated, rising infection and mortality rates in HCWs will paralyse a country's response to COVID-19, and it is bound to have a significant, long-term impact on healthcare delivery, particularly in health systems already grappling with workforce shortage due to lack of trained personnel, skilled labour migration and geographical maldistribution, even prior to pandemic times. ${ }^{14-17}$ Therefore, in order for countries to plan for the present and the future, it is important to record the number of HCWs who have been infected and have died from COVID-19. It is unclear whether data on COVID-19 infection and death among HCWs globally are being collected and published.

As such, the number of COVID-19 infections and deaths among HCWs globally is unknown. The aim of this systematic review was to, therefore, estimate the number of COVID-19 infections and deaths in HCWs in every country in the world as of 8 May 2020, with further demographical analyses where data were available. This will provide a snapshot of the situation for HCWs during the early phases of the pandemic.

\section{METHODS \\ Overview}

A systematic review on the number and proportion of HCWs who have been infected with or died from COVID-19 was conducted as per the published and registered $\operatorname{protocol}^{18}$ (online supplemental appendix S1). The primary outcomes of interest were COVID-19 infections and deaths in HCWs worldwide. Subgroup analyses were performed according to WHO region, country and demographic characteristics. The Preferred Reporting Items for Systematic Review and Meta-Analyses guidelines ${ }^{19}$ were used to report the findings (online supplemental appendix S2).

Due to the time-sensitive nature of the review and the need to report the most up-to-date information for an ever-evolving situation, there were no restrictions on language, information sources used, publication status and types of sources of evidence. Prior to the commencement of this study, all reviewers attended an online training and support session to ensure an accurate and standardised approach to the overall methodological process. Ongoing research support was provided for all collaborators throughout the process.

\section{Search strategy}

Two parallel searches of academic bibliographic databases and grey literature were undertaken.

1. Bibliographic search: the search protocol for this systematic review was executed in MEDLINE and EMBASE, covering the period between the first reported case in the world on 17 November 2019 to 8 May 2020. The search strategy used variants and combinations of search terms related to HCWs and COVID-19 (online 
supplemental appendix S3). The retrieved studies were exported, and duplicate articles were discarded. Two reviewers independently screened the titles and abstracts based on the predefined inclusion and exclusion criteria (online supplemental appendix S1). The full texts of the remaining articles were retrieved and screened by two reviewers independently. Any disagreements were resolved by a third reviewer. The reference lists of all included articles were scrutinised to locate additional relevant publications not identified during the database searches. The reviewers also consulted with senior HCWs-identified and approached through the network of the Oxford University Global Surgery Group-across the world to identify additional publications.

2. Grey literature search: a grey literature search was performed to include sources dedicated to COVID-19 or world data. These sources included government websites, non-governmental websites, social media sites, media websites and preprints on medRxiv. ${ }^{20}$ Snowball searching using a web-based search engine (Google) was used to find additional documents and online sources. A pragmatic approach was taken for the grey literature search and a stepwise guide was provided to all data collectors to ensure consistency of search strategy. A full record of the conducted search is provided in online supplemental appendix S3. The reference list of all included documents identified through the grey literature search was examined to identify any further relevant documents and online sources missed through the above search strategy, until a saturation point was reached where no new sources were identified.

All searches were completed in duplicate by two reviewers independently. A third reviewer validated these searches and resolved any disparities when they arose. Lastly, governments were contacted for further information where possible. The initial search was completed on 22 April 2020. The search was then updated on 8 May 2020 for primary and secondary outcomes.

\section{Data extraction}

A data extraction form (online supplemental appendix S4) was developed to collect the information necessary for data synthesis. This form was piloted by the team before its use. Data extraction was completed in duplicate by two reviewers independently. A third reviewer validated the data extracted and resolved any disagreements. Several data points were extracted, including publication characteristics, country-specific data points, COVID-19-specific data, demographics of affected HCWs and public health measures employed (online supplemental appendix S5). Data from each country were extracted from multiple data sources. Where the same data point was present in more than one data source, the most recently published data source was used to extract that data point. If more than one of these data sources was published on the same date and that date was the most recent, the same data point was extracted from all the data sources in question and compared against one another. Where the data point was identical between the sources, it was included. Where there was a disagreement between the data sources in question about the value of the same data point, only the data point extracted from the data source of the highest quality was included.

\section{Risk of bias assessment}

Two reviewers independently classified the quality of each included document using the AACODS checklist ${ }^{21}$ or the National Institutes of Health (NIH) study quality assessment tools $^{22}$ (online supplemental appendix S6). Any uncertainty regarding the quality of an included document was resolved through discussion among the reviewers.

\section{Synthesis of results}

Mortality and infection numbers have been pooled. HCW infection and deaths due to COVID-19 as a proportion of total population infections and deaths respectively due to COVID-19 have been calculated. HCW deaths due to COVID-19 as a proportion of all HCW COVID-19 infections have also been calculated, to get the case fatality rate (CFR) expressed as number of reported deaths per 100 cases of reported infections.

\section{No patient and public involvement}

This research was done without patient and public involvement. Their input was not sought for the design of the systematic review, the interpretation of the results or the writing or editing of this document.

\section{RESULTS}

\section{Search and selection of studies/sources}

Searches were conducted up to 8 May 2020. The searches yielded a total of 976 potentially relevant citations. After data validation and cleansing, duplicated and irrelevant citations were removed manually. At this point, 594 citations met the eligibility criteria based on our protocol (figure 1).

More specifically for the bibliographic database search, a total of 64 articles were retrieved. Ten duplicates were detected, thus 54 studies remained. After the screening of titles and abstracts based on inclusion and exclusion criteria, 15 of these progressed to full-text screening and 8 studies were included in the final analysis. The grey literature search yielded 912 citations. After screening for inclusion and eligibility, 586 citations remained and were included in the study.

\section{Characteristics of included sources}

Characteristics of the included sources are described in table 1 and figure 2. Overall, 594 records were included, of which 14 were journal articles. Of the remaining records, $19.5 \% \quad(\mathrm{n}=116)$ were government documents, $16.5 \%(\mathrm{n}=98)$ were government websites, $48.3 \% \quad(\mathrm{n}=287)$ were media articles, $9.6 \% \quad(\mathrm{n}=57)$ were research or 


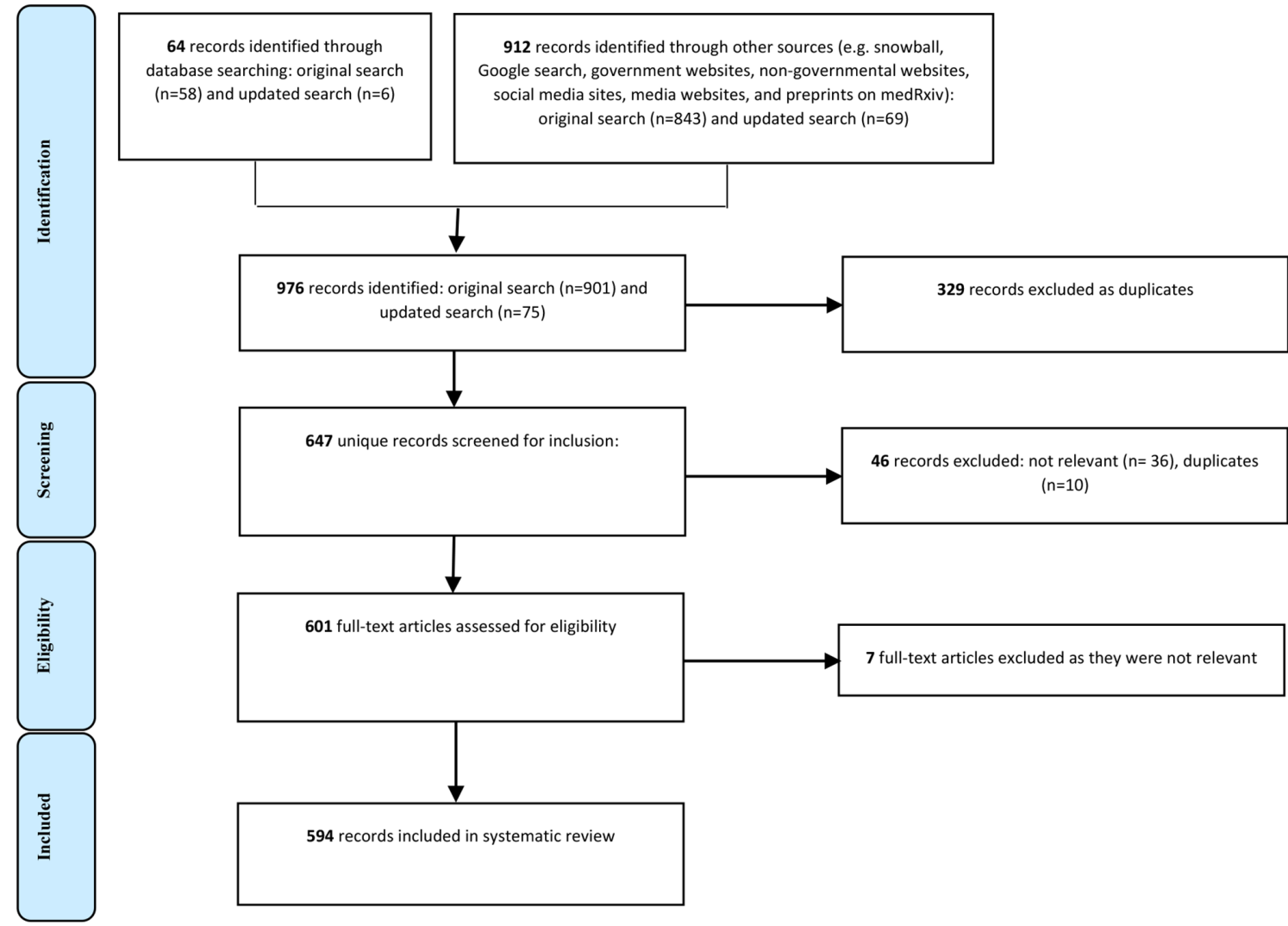

Figure 1 Preferred Reporting Items for Systematic Review and Meta-Analyses flow chart of the source selection process.

academic reports, $3 \%(\mathrm{n}=18)$ were statistical websites and $0.7 \%(\mathrm{n}=4)$ were primary sources. All sources were appraised per the AACODS checklist ${ }^{21}$ or the NIH study quality assessment tools, ${ }^{22}$ as shown in online supplemental appendix S7. Primary outcomes were available for $85.1 \%$ of all countries $(166 / 195)$.

\section{Outcomes}

Primary outcomes

Number of healthcare workers infected with COVID-19 worldwide

As of 8 May 2020, a total of 152888 HCWs had been reported to have been infected with COVID-19 (figure 3). This was $3.9 \%$ of the total number of 3912156 patients with COVID-19 worldwide. It should be noted that 118801 of these patients with COVID-19 were reported in countries where no data on HCWs infections with COVID-19 were available. A total of 130 countries reported at least one case of HCW infection with COVID-19 (figure 3 and online supplemental appendix S8).

\section{Number of healthcare worker deaths with COVID-19 worldwide}

The total number of reported HCW deaths as of 8 May 2020 was 1413 (figure 4). This suggests that for every $100 \mathrm{HCWs}$ that got infected, 1 died. This was also $0.5 \%$ of the total number of 270426 COVID-19 deaths worldwide. It should be noted that 2922 deaths from COVID-19 were reported in countries where no data on HCWs deaths from COVID-19 were available. As of 8 May 2020, 67 countries had reported at least one HCW death related to COVID-19 (figure 4 and online supplemental appendix S8). China and Italy were the first two countries to report HCW deaths, and the first deaths in each of these countries occurred over a month apart (online supplemental appendix S9).

\section{Subgroup analysis \\ Characteristics of healthcare workers who were infected with COVID-19}

Although most countries released the total number of HCW deaths or infections, most published reports did not include details on the age category, ethnicity or role of workers. The data in table 2 are based on a smaller number of countries that made these data available.

The overall median age of the reported HCWs who were infected was 47.3 years (range: $18-84$ ), $71.6 \%$ of whom were women. The overall median age of the HCWs who died was 56.2 years (range: 18-84), $29.2 \%$ of them women. The CFR for men and women were 9.47 and 1.55 , respectively. Nurses were the largest HCW group with COVID-19 infection (38.6\% of those infected). However, doctors were the largest group of HCWs who died (51.4\%). Ethnicity data for deaths were available for Australia, France and the UK and showed 73 deaths in white HCWs and 106 deaths in non-white HCWs. Ethnicity data for infections were only available from the USA and showed 2743 infections in white HCWs and 1058 in non-white HCWs.

\section{Age}

Age-stratified figures were not available for most countries. Data were only available for 14058 of the 152888 


\begin{tabular}{|c|c|c|}
\hline & Number & Percentage (\%) \\
\hline \multicolumn{3}{|l|}{ Publication type } \\
\hline Journal article & 14 & 2.4 \\
\hline Government document & 116 & 19.5 \\
\hline Government websites & 98 & 16.5 \\
\hline Media articles & 287 & 48.3 \\
\hline Research/Academic reports & 57 & 9.6 \\
\hline Statistical websites & 18 & 3.0 \\
\hline Primary sources & 4 & 0.7 \\
\hline $\begin{array}{l}\text { Countries with no data for primary } \\
\text { outcome }\end{array}$ & 29 & 14.9 \\
\hline \multicolumn{3}{|l|}{ Angola } \\
\hline \multicolumn{3}{|l|}{ Barbados } \\
\hline \multicolumn{3}{|l|}{ Belize } \\
\hline \multicolumn{3}{|l|}{ Bolivia } \\
\hline \multicolumn{3}{|l|}{ Cote d'Ivoire } \\
\hline \multicolumn{3}{|l|}{ Djibouti } \\
\hline \multicolumn{3}{|l|}{ Dominica } \\
\hline \multicolumn{3}{|l|}{ Eritrea } \\
\hline \multicolumn{3}{|l|}{ Iraq } \\
\hline \multicolumn{3}{|l|}{ Jordan } \\
\hline \multicolumn{3}{|l|}{ Liechtenstein } \\
\hline \multicolumn{3}{|l|}{ Luxembourg } \\
\hline \multicolumn{3}{|l|}{ Malawi } \\
\hline \multicolumn{3}{|l|}{ Maldives } \\
\hline \multicolumn{3}{|l|}{ Mauritania } \\
\hline \multicolumn{3}{|l|}{ Monaco } \\
\hline \multicolumn{3}{|l|}{ Nicaragua } \\
\hline \multicolumn{3}{|l|}{ North Korea } \\
\hline \multicolumn{3}{|l|}{$\begin{array}{l}\text { North Macedonia (formerly } \\
\text { Macedonia) }\end{array}$} \\
\hline \multicolumn{3}{|l|}{ Norway } \\
\hline \multicolumn{3}{|l|}{ Oman } \\
\hline \multicolumn{3}{|l|}{ Paraguay } \\
\hline \multicolumn{3}{|l|}{ Qatar } \\
\hline \multicolumn{3}{|l|}{ Slovakia } \\
\hline \multicolumn{3}{|l|}{ Solomon Islands } \\
\hline \multicolumn{3}{|l|}{ Syria } \\
\hline \multicolumn{3}{|l|}{ Tonga } \\
\hline Turkmenistan & & \\
\hline United Arab Emirates & & \\
\hline
\end{tabular}

$(9.2 \%)$ reported HCW infection cases and 343 of the $1413(24.3 \%)$ reported HCW deaths as a result of COVID-19. Of all countries, $15 \%$ reported age-related information for COVID-19 cases and $17 \%$ reported information on COVID-19 deaths. The majority of infections was reported in the 50-59 age range. The lowest reported number of infections were in the group aged

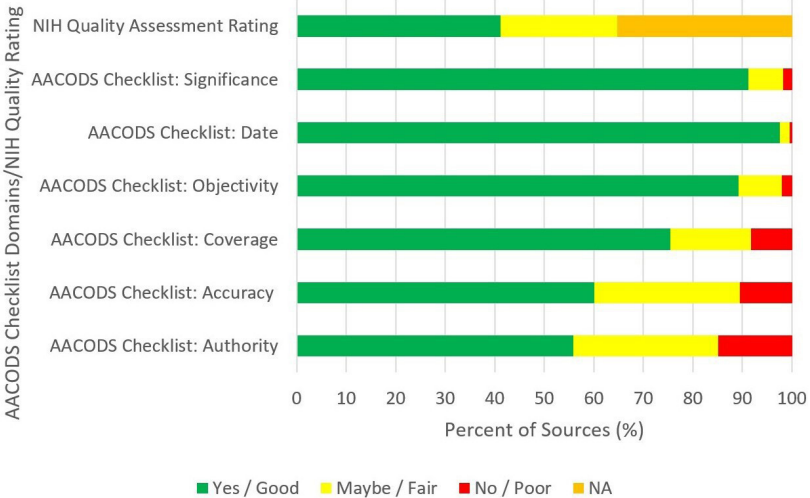

Figure 2 Summary of quality assessments of all 594 sources. NIH, National Institutes of Health.

over 70 years. However, this age group had the highest CFR (table 3). The majority of deaths were also reported in the 50-59 age range, with the lowest number reported in the 18-29 age group.

\section{Specialities}

Speciality-related data were only available from 14 countries: Argentina, Australia, Austria, Bahamas, Cameroon, Canada, China, Colombia, France, Germany, Ghana, Guyana, Turkey and UK (13\% of all data). General practitioners (GPs) appear to be the largest group of doctors who died while mental health nurses constituted the

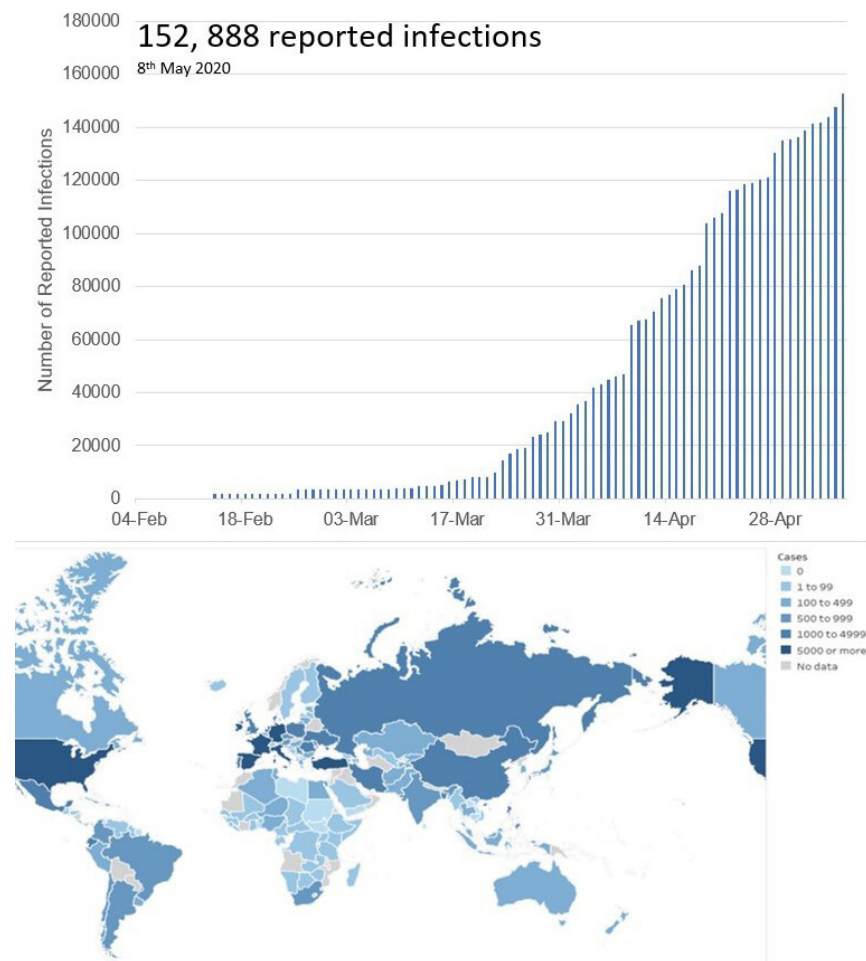

Figure 3 Cumulative number of reported COVID-19 infections in healthcare workers worldwide over time and total number of reported cases of COVID-19 infections in healthcare workers worldwide on 8 May 2020. 


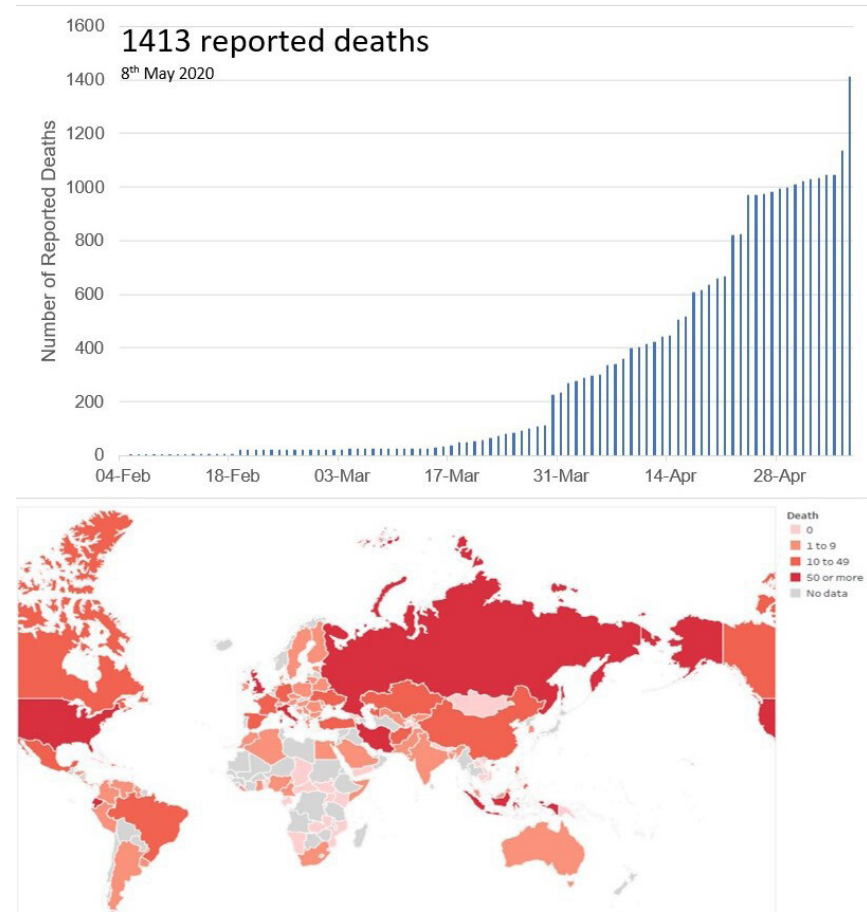

Figure 4 Cumulative number of reported COVID-19 deaths in healthcare workers worldwide and total number of reported cases of COVID-19 deaths in healthcare workers worldwide.

largest group of nurse subspecialists who lost their lives to COVID-19 (table 4 ). There were 30 reported deaths in the UK among doctors, one-third of which were GPs.
Table 3 Distribution of infections, deaths and case fatality in healthcare workers - data do not include cases with unknown age

\begin{tabular}{llcc}
\hline $\begin{array}{l}\text { Age band } \\
\text { (years) }\end{array}$ & Infections & Deaths & CFR $^{*}$ (\%) \\
\hline $18-29$ & 1301 & 14 & 1.1 \\
\hline $30-39$ & 2356 & 20 & 0.8 \\
\hline $40-49$ & 3942 & 45 & 1.1 \\
\hline $50-59$ & 4812 & 123 & 2.6 \\
$60-69$ & 1569 & 112 & 7.1 \\
\hline $70+$ & 78 & 29 & 37.2 \\
\hline
\end{tabular}

${ }^{*} \mathrm{CFR}$ is the number of reported deaths per 100 cases of reported infections.

CFR, case fatality rate.

\section{WHO regions}

The highest number of COVID-19 infections of HCWs were reported in Europe (119 628, 78.2\%), while the lowest number was reported in Africa (1472, 1.0\%) (figure 5). The same regional pattern was observed regarding deaths: Europe reported the highest number of deaths $(712,50.4 \%)$ and Africa the lowest $(17,1.2 \%)$ (figure 5). Although the highest number of deaths were reported in Europe, the number of infections was also so large that Europe was the region reporting the lowest CFR. The highest CFR is seen in the Eastern Mediterranean region ( 5.7 deaths per 100 infections), followed by South East Asia (3.1 deaths per 100 infections) (table 5).

Table 2 Characteristics of healthcare workers with COVID-19 infection and deaths

\begin{tabular}{|c|c|c|}
\hline Characteristic & Infection & Death \\
\hline \multirow[t]{2}{*}{ Age $^{i}$} & Median age: 47.3 years $(n=14058)$ & Median age: 56.2 years $(n=343)$ \\
\hline & Range: $18-84$ years & Range: $18-84$ years \\
\hline Sex ${ }^{i i}$ & Female: $71.6 \%(n=14656)$ & Female: $29.2 \%(n=227)$ \\
\hline \multirow[t]{3}{*}{ Level (number) ${ }^{\text {iii. }}$} & Student: $<0.1 \%(n=13)$ & Student: $0.3 \%(n=1)$ \\
\hline & Qualified: $99.9 \%(n=36081)$ & Qualified: $96.4 \%(n=350)$ \\
\hline & Retired: $<0.1 \%(n=10)$ & Retired: $2.7 \%(n=10)$ \\
\hline \multirow[t]{3}{*}{ Type of HCW iv. } & Doctor: $31.3 \%(n=8688)$ & Doctor: $51.4 \%(n=525)$ \\
\hline & Nurse: $38.6 \%(n=10706)$ & Nurse: $25.3 \%(n=259)$ \\
\hline & Administrators: $<0.1 \%(n=29)$ & Administrators: $2.8 \%(n=29)$ \\
\hline \multirow[t]{2}{*}{ Admission to intensive care unit ${ }^{v}$. } & $\mathrm{n}=1158$ & NA \\
\hline & $\begin{array}{l}\text { i. Data from } 15 \% \text { of all countries; ii. Data } \\
\text { from } 21 \% \text { of all countries; iii. Data from } 7 \% \\
\text { of all countries; iv. Data from } 13 \% \text { of all } \\
\text { countries; v. Data from } 10 \% \text { of all countries }\end{array}$ & $\begin{array}{l}\text { i. Data from } 17 \% \text { of all countries; ii. Data } \\
\text { from } 21 \% \text { of all countries; iii. Data from } \\
13 \% \text { of all countries; iv. Data from } 22 \% \text { of } \\
\text { all countries. }\end{array}$ \\
\hline
\end{tabular}

HCW, healthcare worker; NA, not available. 


Table 4 Healthcare worker mortality by subspecialty (data
available for only $13 \%$ of all deaths)

\begin{tabular}{lll}
\hline Nurses & Medicine: 15 & COVID-19 ward 4 \\
& Intensive care units 3 \\
& Acute admission 3 \\
& Adult care 2 \\
& Palliative 2 \\
& Cardiology 1 \\
& Orthopaedics 1 \\
& Mental health 14 \\
Surgery: 1 & Care home 8 \\
Other: 26 & Community 2 \\
& Dental 1 \\
& Disability 1
\end{tabular}

\begin{tabular}{|c|c|c|}
\hline Doctors & Medicine: 45 & $\begin{array}{l}\text { General practitioner } 18 \\
\text { Emergency medicine } 5 \\
\text { Internal medicine } 4 \\
\text { Paediatrics } 3 \\
\text { Geriatrics } 3 \\
\text { Neurology } 2 \\
\text { Pathologist } 2 \\
\text { Haem-oncology } 1 \\
\text { Infectious disease } 1 \\
\text { Microbiology } 1 \\
\text { Nephrology } 1 \\
\text { Psychiatry } 1 \\
\text { Respiratory } 1 \\
\text { Anaesthetics } 1 \\
\text { Gastroenterology } 1\end{array}$ \\
\hline & Surgery: 14 & $\begin{array}{l}\text { Obstetrics and Gynaecology } 3 \\
\text { Ophthalmology } 2 \\
\text { Ear, Nose and Throat } 2 \\
\text { Urology } 2 \\
\text { Cardiothoracic } 1 \\
\text { Endocrine surgery } 1 \\
\text { General surgery } 1 \\
\text { Orthopaedics } 1 \\
\text { Vascular } 1\end{array}$ \\
\hline & Other: 6 & $\begin{array}{l}\text { Director } 2 \\
\text { Public health } 1 \\
\text { Unknown } 3\end{array}$ \\
\hline
\end{tabular}

\section{Countries}

On 8 May 2020, Spain reported the highest cumulative number of COVID-19 infections in HCWs in the world at 30663 (20\% of all HCW infections). This is followed closely by Italy (23718) and the Netherlands (13884). Italy reported the highest cumulative number of deaths in HCWs due to COVID-19 at 220 (figure 6). At least 10\%

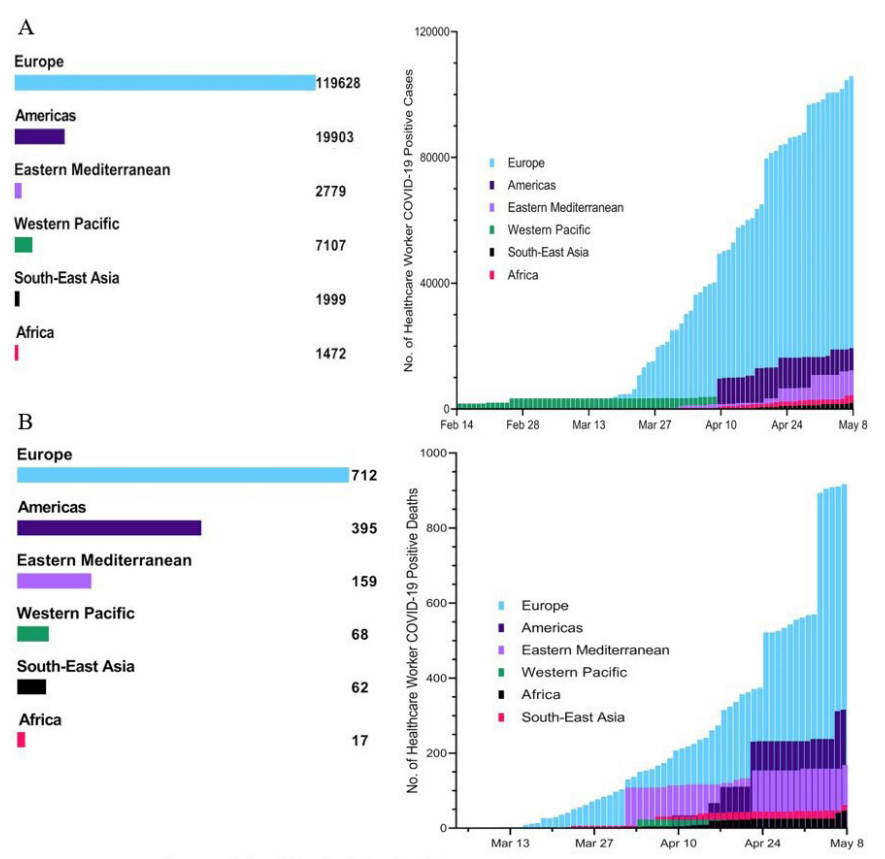

Figure 5 COVID-19 infections (A) and deaths (B) in healthcare workers in $\mathrm{WHO}$ regions.

of all COVID-19 deaths were among healthcare workers in five countries: Guyana, Venezuela, Afghanistan, Costa Rica and Kazakhstan. Full numbers and CFRs for all countries can be found in online supplemental appendices S8 and S9. The COVID-19 infection peak varies from country to country with China and Italy demonstrating some of the earliest peaks. HCW infections and deaths reflect this as can be seen in online supplemental appendix S9. Some countries are only at the early stages of COVID-19 infection.

\section{DISCUSSION}

Here, we present the first systematic review to date examining the number of COVID-19 infections and deaths among HCWs across 195 countries during the early phases of the pandemic, when the USA and Europe were thought to be the epicentres of the pandemic. We conducted this search to estimate the infection and mortality burden among all individuals involved in the care of patients with COVID-19-from diagnosis to treatment and rehabilitation. In addition, we hoped to identify any factors associated with the risk of infection and death in HCWs.

There is an important need to address the incidence of COVID-19-related illness in HCWs globally. Failure to address infection and mortality among HCWs has the potential to further increase transmission of COVID-19 within healthcare facilities and their wider communities. $^{23} 24$ The resulting shortage of HCWs may impair the quality of the provision of health services nationally both during the acute phase of the pandemic, and in the long term. Occupational risks in the workplace must be minimised if not altogether eliminated. Moreover, a clear 
Table 5 Total number of reported infections and deaths in WHO regions

\begin{tabular}{lccll}
\hline WHO region & Infections & Deaths & CFR $^{\star}$ (\%) & $\begin{array}{l}\text { Healthcare workers COVID-19 deaths/ } \\
\text { Total population COVID-19 deaths (\%) }\end{array}$ \\
\hline Africa & 1472 & 17 & 1.2 & 0.06 \\
Eastern Mediterranean & 2779 & 159 & 5.7 & 0.44 \\
\hline Europe & 119628 & 712 & 0.6 & 1.40 \\
Americas & 19903 & 395 & 2.0 & 4.58 \\
\hline South-East Asia & 1999 & 62 & 3.1 & 0.20 \\
Western Pacific & 7107 & 68 & 1.0 & 0.06 \\
\hline Total & 152888 & 1413 & 0.92 & 0.52 \\
\hline
\end{tabular}

${ }^{*} \mathrm{CFR}$ is the number of reported deaths per 100 cases of reported infections.

CFR, case fatality rate.

pathway must be present for the early diagnosis and treatment of HCWs suspected to have contracted COVID-19. It is essential that measures are put in place to ensure that HCWs are continually protected.

\section{Key findings}

A total of 152888 infections and 1413 deaths were reported. The overall infection and death trends among HCWs followed that of the general population. Infections were mainly in women, but deaths were mainly in men. Infections were seen more in nurses, deaths more in doctors. Within the data available, GPs were the highest risk specialty for deaths among doctors, while the highest risk nursing specialty was mental health. It is possible that there was less PPE available in the community, with confirmed cases in hospital wards being

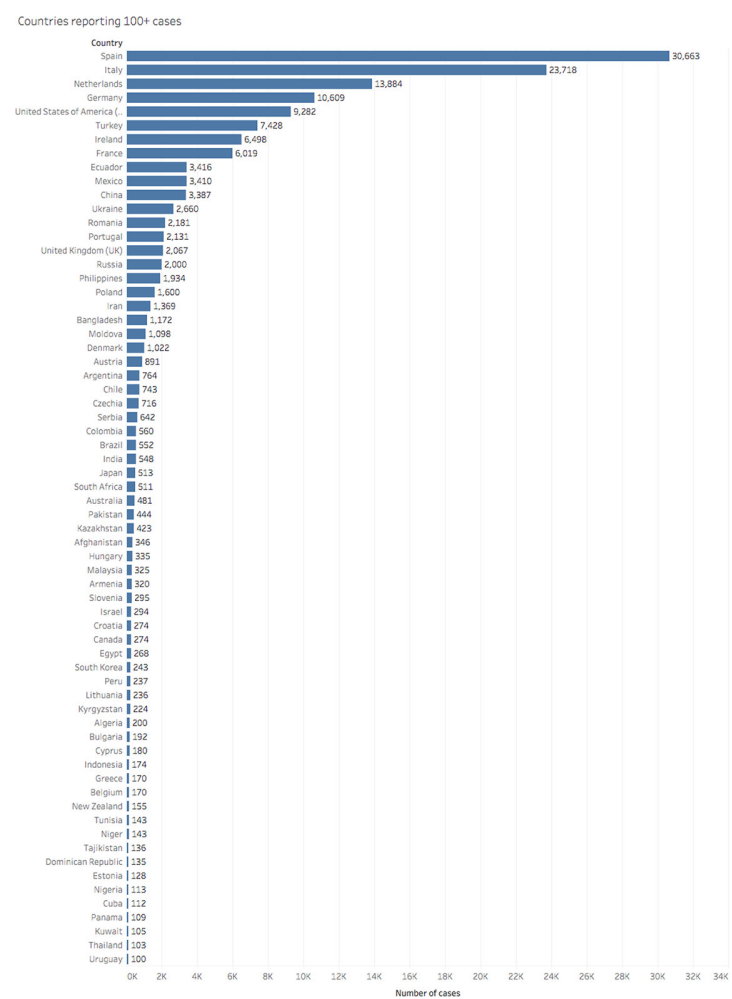

prioritised, or that the high flow of patients through GP services has led to an increased risk of viral transmission. It may just reflect the higher number of GPs compared with hospital doctors. Mental health specialists may also be lacking PPE. ${ }^{25}$ Many mental health nurses also work in the community and often work in close proximity to patients, which may increase their risk of exposure. The majority of infections and deaths were reported in the 50-59 age range, while the group aged over 70 years had the highest CFR. Europe had the highest number of infections and deaths, but the lowest CFR, while the Eastern Mediterranean region had the highest CFR. By population, regions such as the Indian subcontinent and Africa reported relatively low numbers of HCW infection and death.

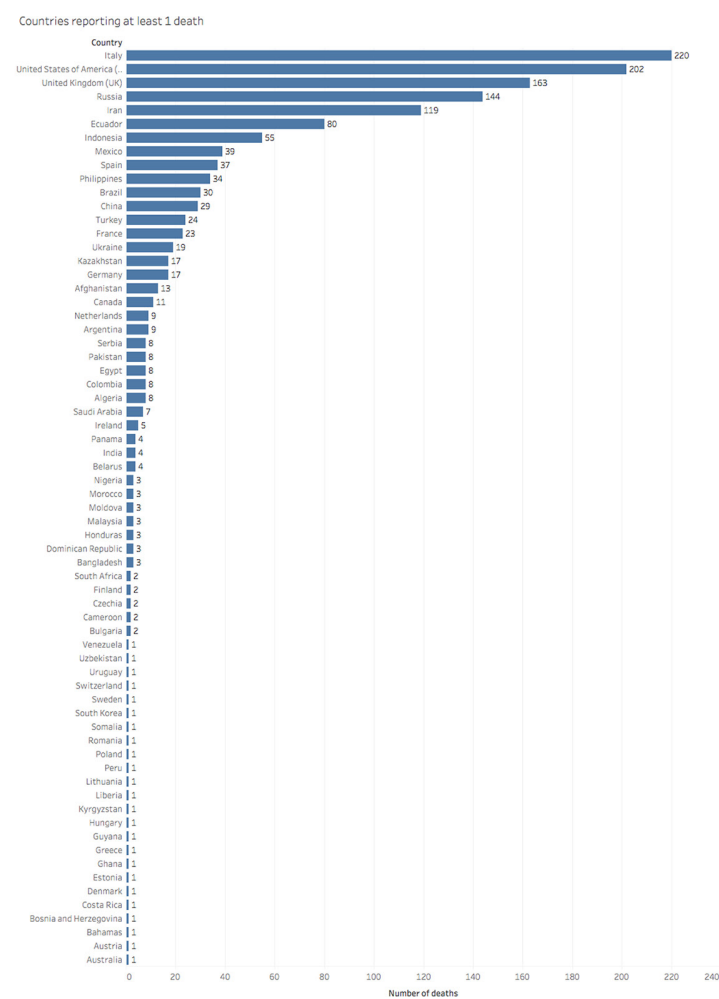

Figure 6 Number of reported healthcare worker infections and deaths due to COVID-19 per country up to 8 May 2020. 
These trends must be considered in the context of the paucity of data, and the natural history of the disease. The differing COVID-19 infection curves in different countries is evident in the reporting trends; some countries were at the beginning of the HCW infection peak, while some were beyond. The first reported case of COVID-19 in Africa was nearly a month after the first case in Europe, and Africa was slightly behind Europe in its disease course; their lower reported numbers of HCW infection is therefore unsurprising, as they are also reporting lower rates of infection overall. If this continues as the epidemic in Africa progresses, it will be necessary to consider what lessons can be learnt. Furthermore, reporting varies significantly between countries. The USA, one of the countries most severely affected by the pandemic, had not reported data about this topic for over 1 month. While other countries, such as the Philippines, provide daily updates. The availability and quality of testing and guidelines for COVID-19 testing varies greatly across countries, which further limits the reliability of the observed trends. Estimating the percentages of HCWs infected by COVID-19 is crucial for adjusting infection prevention policies applied in the healthcare system to reduce viral transmission.

\section{Limitations}

The primary limitation of this systematic review was the quality of the data available to us. A wide range of data was used, including grey literature, which made it difficult to normalise datasets. Furthermore, different countries were at different stages of their epidemics when we collected data. Data from countries at different stages of their epidemics are not comparable. To make data comparable between different countries we would have needed to batch them according to when their epidemics started, but clear information about this was unavailable. As countries move past the peak of the virus and life begins to move back to normality, increased availability of high-quality data should allow us to conduct more extensive quantitative analysis of HCW infections. A retrospective analysis would allow countries to be matched at the same stage of the pandemic - thus allowing a like-for-like comparison.

For our primary analyses, a key limitation was the heterogeneity in HCW classification. Differences here made it difficult to accurately compare data between the countries because, for example, some countries may include all allied healthcare professionals in their numbers, others may not, which could result in reporting inaccurate proportions of HCWs infected by COVID19. Additionally, there was limited access to accurate data about confounding variables, such as availability of testing for COVID-19 in different countries, which could have influenced infection and mortality rates among HCWs. Due to lack of testing, many cases of COVID-19 are diagnosed as 'atypical pneumonia' in some countries and thus do not feature in published figures for COVID-19 cases or deaths. Given the lack of robust data across different variables, including confounding factors, it was not possible to establish causative or even correlative links between the different variables collected and we were, therefore, limited to descriptive analyses.

\section{Recommendations}

COVID-19 infections and deaths among HCWs necessitate provision of adequate and appropriate PPE. Infection control training must be provided for those working on the frontlines of the COVID-19 outbreak response, especially among redeployed HCWs with little experience in the clinical management of infectious diseases. ${ }^{26}$ Regulative and supportive measures must be put into place to ensure compliance with infection control policies in the workplace at all times.

The first step to achieve this would involve appropriate measures for identifying and registering those who have been infected as soon as possible (box 1). Our study clearly highlights the lack of universal access to early identification measures and infection registration processes across healthcare systems in the world during the early phases of the pandemic. The testing guidelines, access and reporting systems vary hugely across countries and are not merely a reflection of country level healthcare expenditure, although this is an important factor and further highlights inequalities between high-income countries (HICs) and low-income and middle-income countries (LMICs). The unavailability of relevant data in a timely manner (which was seen in both HICs and LMICs) makes it difficult to estimate the true burden of infection and effectively plan management strategies. It also inhibits an attempt to learn from those countries beyond their peak and plan timely preventative measures in those who are yet to experience the peak. We highly recommend universal guidelines to be in place for testing and reporting of infections in HCWs in a timely manner, with consideration towards an international HCW infection registry .

The gender-related difference in infection and death rates in HCWs is one that has not been reported previously. Many factors may contribute towards this including more nursing staff being female and more doctors being male, which may reflect differences in exposure levels, training and equipment provided or age at qualification. Further investigation of the identified trends would be recommended.

Although physicians working in certain specialities may be considered high-risk due to frequent exposure to oronasal secretions (eg, otolaryngology, anaesthesiology, dentistry), ${ }^{27}$ the risk to other specialties who work in other healthcare settings, including clinics and mental health facilities, must not be underestimated. The high rate of morbidity and mortality in elderly HCWs may require assigning them to less risky settings such as telemedicine, non-COVID-19 outpatient clinics or administrative positions. ${ }^{28}$ HCWs who report possible symptoms and those who have had unprotected exposure to patients with COVID-19 must be prioritised for testing. HCWs must 


\section{Box 1 Implications for policy and practice}

Protecting all healthcare workers (HCWs) from infection and mortality must be a core element of any pandemic response. It is essential for the welfare of the HCWs themselves as well as healthcare systems more generally because of the critical role HCWs play during pandemic responses - an increase in HCW infection and mortality puts patients at risk, increases the burden on non-infected HCWs and will generally impede safe, efficient and effective delivery of highquality care.

Learning from the COVID-19 pandemic has, so far, pointed to important lessons that need to be addressed in the next 6-12months to prepare for potential second waves of COVID-19 and which will also help to build the general resilience of pandemic responses.

- Measures must be put in place to ensure that all HCWs on the frontline of pandemic response are continually protected:

- Infection control training must be provided for those working on the frontline of the pandemic response, especially among redeployed HCWs with limited experience in the clinical management of infectious diseases.

- Regulative and supportive measures must be put into place to ensure compliance with infection control policies in the workplace at all times.

- Adequate supply chains, and stores of personal protective equipment (PPE) must be ensured.

- Adequate and fair distribution mechanisms must be implemented to ensure healthcare organisations can efficiently get the PPE needed for their HCWs.

- A clear pathway must be present for the early diagnosis, protection and treatment of HCWs suspected to have been infected:

- Mechanisms must be put in place to ensure the latest guidelines on diagnosis are implemented. Additionally, adequate supply chains and distribution mechanisms to procure and distribute, respectively, diagnostics efficiently must be arranged.

- Pathways to ensure early identification and registration of infected HCWs should be implemented.

- Pathways must be put in place to ensure infected HCWs are isolated to prevent them from spreading the infection.

- Treatment based on current best practice for the infection should be delivered to infected HCWs.

- Vulnerable HCWs at high risk of infection/mortality should be given less risky deployments (eg, telemedicine consults, non-COVID-19 outpatient clinics or administrative positions) whenever possible. If this is not possible, they should be prioritised for protective measures and early testing.

- HCWs should be offered flexible working hours to avoid overwork, and psychological intervention plans must be implemented to help HCWs in coping with physical and psychological stress.

In the medium to long term, we highly recommend universal guidelines be designed and implemented for HCW classification as well as testing and reporting of infections in HCWs in a timely manner, with consideration towards an international HCW infection registry to facilitate estimations of the true burden of infection, which can inform the design and implementation of effective management strategies. To promote equity across high-income countries and low-income and middle-income countries, we recommend international agreements be put in place on the equitable distribution of accessible knowledge, PPE, diagnostics and treatment as well as data collection and analytic capacity.

be offered flexible working hours to avoid overwork, and psychological intervention plans must be implemented to help HCWs in coping with physical and psychological stress. $^{29}$
Despite the limitations, our analyses do provide a broad coverage of the data available across the world. The data were run through risk of bias assessments to ensure that an acceptable standard across all datasets was maintained, so that we could compare them. The descriptive analyses also importantly point to the lack of reliable data in so many countries due to lack of infrastructure to quickly and robustly capture data on HCWs and other aspects of healthcare systems that could affect COVID-19-related morbidity and mortality among them. The countries whose datasets had a low risk of bias could serve as examples and provide best practice for countries lacking robust data collection policies and data collection systems. Our pragmatic approach in this study provides general trends to provide rapid information in response to widespread urgent calls from HCWs worldwide.

\section{Author affiliations}

${ }^{1}$ Oxford University Global Surgery Group, Nuffield Department of Surgical Sciences, University of Oxford, Oxford, Oxfordshire, UK

${ }^{2}$ Philippine General Hospital, University of the Philippines Manila College of Medicine, Manila, Metro Manila, The Philippines

${ }^{3}$ Kasr Al Ainy School of Medicine, Cairo University Kasr Alainy Faculty of Medicine, Cairo, Egypt

${ }^{4}$ Department of Surgery, University of Nairobi College of Health Sciences, Nairobi, Kenya

${ }^{5}$ Faculty of Pharmacy, Al Azhar University-Gaza, Gaza, State of Palestine

${ }^{6}$ Dipartimento di medicina clinica e sperimentale, University of Insubria, Varese, Lombardia, Italy

${ }^{7}$ University of Rwanda College of Medicine and Health Sciences, Kigali, Rwanda

${ }^{8}$ North Bristol NHS Trust, Westbury on Trym, UK

${ }^{9}$ Mbarara University of Science and Technology, Mbarara, Mbarara, Uganda

${ }^{10}$ Islamic University of Gaza, Gaza, State of Palestine

${ }^{11}$ Shriners Ambulatory Clinic, Tijuana, Mexico

${ }^{12}$ Department of Plastic Surgery, Hospital General Dr. Manuel Gea Gonzalez,

National Autonomous University of Mexico, Mexico City, Mexico

${ }^{13}$ Palestinian Ministry of Health, Gaza, State of Palestine

${ }^{14}$ University of California San Diego, La Jolla, California, USA

${ }^{15}$ Gelephu Central Regional Referral Hospital, Gelephu, Bhutan

${ }^{16}$ Sheikh Hasina National Institute of Burn and Plastic Surgery, Dhaka, Bangladesh

${ }^{17}$ National University of Science and Technology Faculty of Medicine, Bulawayo,

Zimbabwe

${ }^{18}$ Gulu University Faculty of Medicine, Gulu, Uganda

${ }^{19}$ Oxford Brookes University, Oxford, UK

${ }^{20}$ Swansea Bay University Health Board, Port Talbot, UK

${ }^{21}$ University of Huddersfield, Huddersfield, UK

${ }^{22}$ Harvard Medical School, Boston, Massachusetts, USA

${ }^{23}$ ConnectMed International, Dominican Republic, Dominican Republic

${ }^{24}$ Instituto Mexicano del Seguro Social, Ciudad de Mexico, Mexico

${ }^{25}$ Jinnah Post Graduate Medical Centre, Karachi, Pakistan

${ }^{26}$ Lancaster Medical School, Lancaster University, Lancaster, UK

${ }^{27}$ Nuffield Department of Orthopaedics, Rheumatology, and Musculoskeletal

Sciences, Oxford University, Oxford, UK

${ }^{28}$ Parirenyatwa Hospital, University of Zimbabwe College of Health Sciences, Harare, Zimbabwe

${ }^{29}$ Department of Surgery, University of Alberta, Edmonton, Alberta, Canada

${ }^{30}$ Office of Global Surgery, University of Alberta, Edmonton, Alberta, Canada

${ }^{31}$ Centre for Statistics in Medicine, University of Oxford, Oxford, UK

${ }^{32}$ MRC Lifecourse Epidemiology Unit, Southampton General Hospital, University of Southampton, Southampton, UK

Twitter Soham Bandyopadhyay @SohamBGlobal, Ronnie E Baticulon @ronibats, Zoe Li @Zwl21, 0said Alser @0saidalserMD, Rafael Pinedo-Villanueva @RafaPinedo_HE and Roba Khundkar @robakhundkar 
Acknowledgements The authors would like to thank Professor Kokila Lakhoo (Nuffield Department of Surgical Sciences, University of Oxford, UK) for her advice that helped refine the aims and objectives of this article.

Contributors RK is the guarantor. RK and SB conceived the project. SB, RK, and AJ contributed equally to the design of the project. All authors contributed to the collection of the data. SB, REB, MA, DKO, YB, AK, SAP, GB, DK, SC, MK, AJ, AM and RK drafted the manuscript. All remaining authors extensively reviewed the manuscript. All authors have approved the final manuscript and are willing to take responsibility for appropriate portions of the content.

Funding The authors have not declared a specific grant for this research from any funding agency in the public, commercial or not-for-profit sectors.

Map disclaimer The depiction of boundaries on this map does not imply the expression of any opinion whatsoever on the part of BMJ (or any member of its group) concerning the legal status of any country, territory, jurisdiction or area or of its authorities. This map is provided without any warranty of any kind, either express or implied.

\section{Competing interests None declared.}

Patient consent for publication Not required.

Provenance and peer review Not commissioned; externally peer reviewed.

Data availability statement Data are available on request. All data relevant to the study are included in the article or uploaded as supplementary information. Any further data are available on request.

Supplemental material This content has been supplied by the author(s). It has not been vetted by BMJ Publishing Group Limited (BMJ) and may not have been peer-reviewed. Any opinions or recommendations discussed are solely those of the author(s) and are not endorsed by BMJ. BMJ disclaims all liability and responsibility arising from any reliance placed on the content. Where the content includes any translated material, BMJ does not warrant the accuracy and reliability of the translations (including but not limited to local regulations, clinical guidelines, terminology, drug names and drug dosages), and is not responsible for any error and/or omissions arising from translation and adaptation or otherwise.

Open access This is an open access article distributed in accordance with the Creative Commons Attribution Non Commercial (CC BY-NC 4.0) license, which permits others to distribute, remix, adapt, build upon this work non-commercially, and license their derivative works on different terms, provided the original work is properly cited, appropriate credit is given, any changes made indicated, and the use is non-commercial. See: http://creativecommons.org/licenses/by-nc/4.0/.

\section{ORCID iDs}

Soham Bandyopadhyay http://orcid.org/0000-0001-6553-3842

Zoe Li http://orcid.org/0000-0003-1129-3200

Osaid Alser http://orcid.org/0000-0001-6743-803X

\section{REFERENCES}

1 World Health Organization. WHO Director-General's opening remarks at the media briefing on COVID-19. Available: https://www.who.int/ $\mathrm{dg} /$ speeches/detail/who-director-general-s-opening-remarks-at-themedia-briefing-on-covid-19-11-march-2020 [Accessed 3 May 2020].

2 Zhu N, Zhang D, Wang W, et al. A novel coronavirus from patients with pneumonia in China, 2019. N Engl J Med 2020;382:727-33.

3 World Health Organization. Coronavirus disease, 2019. Available: https://www.who.int/emergencies/diseases/novel-coronavirus-2019 [Accessed 3 May 2020].

4 Chen Q, Liang M, Li Y, et al. Mental health care for medical staff in China during the COVID-19 outbreak. Lancet Psychiatry 2020;7:e15-16.

5 Lai J, Ma S, Wang Y, et al. Factors associated with mental health outcomes among health care workers exposed to coronavirus disease 2019. JAMA Netw Open 2020;3:e203976.
6 Greenberg N, Docherty M, Gnanapragasam S, et al. Managing mental health challenges faced by healthcare workers during covid-19 pandemic. BMJ 2020;368:m1211

7 World Health Organization. Health workers: a global profile, 2020. Available: https://www.who.int/whr/2006/06_chap1_en.pdf

8 World Health Organization. Rational use of personal protective equipment for coronavirus disease (COVID-19) and considerations during severe shortages. Available: https://www.who.int/ publications-detail/rational-use-of-personal-protective-equipmentfor-coronavirus-disease-(covid-19)-and-considerations-duringsevere-shortages

9 Forrester JD, Nassar AK, Maggio PM, et al. Precautions for operating room team members during the COVID-19 pandemic. $J$ Am Coll Surg 2020;230:1098-101.

10 Bhangu A, Lawani I, Ng-Kamstra JS, et al. Global guidance for surgical care during the COVID-19 pandemic. Br J Surg 2020

11 Dashraath P, Wong JLJ, Lim MXK, et al. Coronavirus disease 2019 (COVID-19) pandemic and pregnancy. Am J Obstet Gynecol 2020;222:521-31.

12 Cook TM, El-Boghdadly K, McGuire B, et al. Consensus guidelines for managing the airway in patients with COVID -19. Anaesthesia 2020;75:785-99.

13 Razai MS, Doerholt K, Ladhani S, et al. Coronavirus disease 2019 (covid-19): a guide for UK GPs. BMJ 2020;368:m800.

14 Sidibé M, Campbell J. Reversing a global health workforce crisis. Bull World Health Organ 2015;93:3.

15 Liu JX, Goryakin Y, Maeda A, et al. Global health workforce labor market projections for 2030. Hum Resour Health 2017;15.

16 Global Health Workforce Alliance, World Health Organization. A universal truth: no health without a workforce, 2014.

17 World Health Organization. Global strategy on human resources for health: workforce, 2030.

18 Bandyopadhyay S, Aggarwal A, Kamath A, et al. Infection and mortality of healthcare workers worldwide from COVID-19: a scoping review. Infect Dis 2020.

19 Moher D, Liberati A, Tetzlaff J, et al. Preferred reporting items for systematic reviews and meta-analyses: the PRISMA statement. PLoS Med 2009;6:e1000097.

20 medRxiv. medRxiv.org - the preprint server for Health Sciences, 2020. Available: https://www.medrxiv.org/

21 Tyndall J. AACODS Checklist, 2010. Available: https://dspace. flinders.edu.au/xmlui/bitstream/handle/2328/3326/AACODS Checklist.pdf;jsessionid=033717B9674D1B6789BA829F099C8D33? sequence $=4$

22 National Heart Lung and Blood Institute. Study quality assessment tools, 2020. Available: https://www.nhlbi.nih.gov/health-topics/ study-quality-assessment-tools

23 Contagion Live. Coronavirus: for health care workers, risk of infection, but also burnout, 2020. Available: https://www. contagionlive.com/news/for-health-care-workers-risk-of-infectionbut-also-burnout

24 The Guardian. Healthcare workers 'should be screened for Covid-19 every week', 2020. Available: https://www.theguardian.com/world/ 2020/apr/16/healthcare-workers-screened-covid-19-every-weekinfectious-unethical

25 NHS Providers. Mental health PPE and testing concerns must be addressed, 2020. Available: https://nhsproviders.org/newsblogs/news/mental-health-ppe-and-testing-concerns-must-beaddressed

26 Liu Q, Luo D, Haase JE, et al. The experiences of health-care providers during the COVID-19 crisis in China: a qualitative study. Lancet Glob Health 2020;8:e790-8.

27 Edsel B, Xu A, Salimi A, et al. MD death COVID-19. medRxiv 2020.

28 CDC COVID-19 Response Team. Characteristics of Health Care Personnel with COVID-19 - United States, February 12-April 9, 2020. MMWR Morb Mortal Wkly Rep 2020;69:477-81.

29 Wang C, Pan R, Wan X, et al. A longitudinal study on the mental health of general population during the COVID-19 epidemic in China. Brain Behav Immun 2020;87:40-8. 